\title{
ACOUSTIC PROPERTIES OF MAGNETIC FLUIDS BASED ON TRANSFORMER OIL UNDER MAGNETIC FIELD
}

\author{
Jozef Kúdelčík ${ }^{*}$ - Peter Bury ${ }^{*}$ - Peter Kopčanský ${ }^{* *}$ \\ — Milan Timko ${ }^{* *}$ — Vlasta Závišová ${ }^{* *}$
}

\begin{abstract}
The structural changes in magnetic fluids based on transformer oil TECHNOL and MOGUL upon the effect of an external magnetic field and temperature were studied by acoustic spectroscopy. When a magnetic field is increased, the interaction between the magnetic field and the magnetic moments of the nanoparticles leads to the aggregation of magnetic nanoparticles and following clusters formation. However, the temperature of magnetic fluids has also very important influence on the structural changes because of the mechanism of thermal motion that acts against the cluster creation. The live time of clusters have relative long time scale for the magnetic fluid based on TECHNOL, while for MOGUL is quite short.
\end{abstract}

K e y w ords: magnetic fluid, transformer oil, acoustic spectroscopy, clusters, magnetic field

\section{INTRODUCTION}

The transformer oil based magnetic fluids are colloidal suspensions of magnetic nanoparticles covered with a surfactant layer in transformer oils $[1,2]$. The transformer oil with magnetic nanoparticles that is usually used for both high voltage insulation and power electronics cooling is subjected to research to enhance its characteristics. Such magnetic fluids should have better insulating and thermal properties. The dielectric breakdown strength of transformer oil, however, is strongly influenced by the aggregation effects of magnetic nanoparticles and can induce electric breakdown $[3,4]$.

One of the methods of studying changes in the magnetic fluid structure under the magnetic field and influenced by temperature is based on the measurements of changes in the acoustic wave attenuation $\alpha$ [5-7]. The change of the acoustic attenuation of acoustic waves propagating through suspensions, in which magnetic nanoparticles constituting one phase are dispersed in a continuous second phase, can indicate characterized properties and structure of magnetic liquids. The interaction between the acoustic waves and the magnetic nanoparticles or their aggregation to clusters leads to additional attenuation of acoustic wave compared to that in the carried liquid.

Under the effect of an external magnetic field the nanoparticles of magnetic fluid become arranged into oligomers or clusters, forming chains stiffening the liquid structure. Acoustic wave propagation in magnetic fluid placed in magnetic field was studied by several authors both theoretically and experimentally $[2,5,6]$. There are also computer simulations using Monte Carlo $[8,9]$ investigating aggregation phenomena in a polydisperse colloidal dispersion of ferromagnetic nanoparticles. All these works suppose that chainlike clusters are formed along magnetic field direction, but clusters can have various shapes. These shapes depend on both particle-particle and particle-field interactions. In this paper the interference of magnetic field, time and temperature on the structure of magnetic fluids based on transformer oils MOGUL and TECHNOL with similar concentration is presented. The structure changes are studied by acoustic spectroscopy, observed results for both kinds of magnetic fluids are discussed and compared.

\section{EXPERIMENTAL RESULTS}

The block diagram of the experimental arrangement is shown in Fig. 1. The measurements of the attenuation of the acoustic wave of frequency $12.4 \mathrm{MHz}$ were carried out by a pulse method using the acoustic equipment EXPLORER II NDT WORKSTATION, (MATEC). An acoustic wave generated by transducer, propagated through the magnetic fluids placed in the thermostatted closed measuring cell $\left(1.5 \times 0.9 \times 1.0 \mathrm{~cm}^{3}\right.$, the temperature was stabilized with an accuracy $\pm 0.2{ }^{\circ} \mathrm{C}$ ) inserted in an electromagnet underwent a multiple reflection between transducers. The first two selected adjacent echoes representing different paths after reflection and reaching a receiving transducer were received by MATEC. The computer program controlled the whole experiment, the increase and decrease rate of magnetic field, records the time developments of acoustic signal, calculated the acoustic attenuation at given magnetic fluid and stored observed result.

The subjects of the study were magnetic fluids based on transformer oils TECHOL and MOGUL. The magnetic fluids (MF) used in experiments consisted of magnetite nanoparticles (FeO.Fe2O3) with the mean diameter $d \approx 11 \mathrm{~nm}$, coated with oleic acid as a surfactant

\footnotetext{
* Department of Physics, Žilinská univerzita v Žiline, Univerzitná 1, 01026 Žilina, Kudelcik@fyzika.uniza.sk; ${ }^{* *}$ Department of Magnetism, IEP SAS, Watsonova 47, 04001 Košice
} 


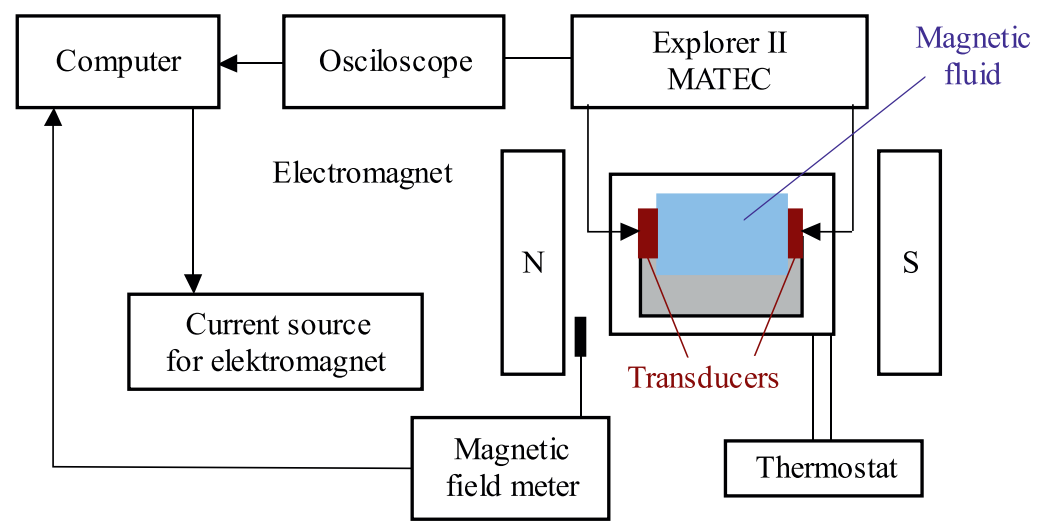

Fig. 1. Block diagram of experimental arrangement

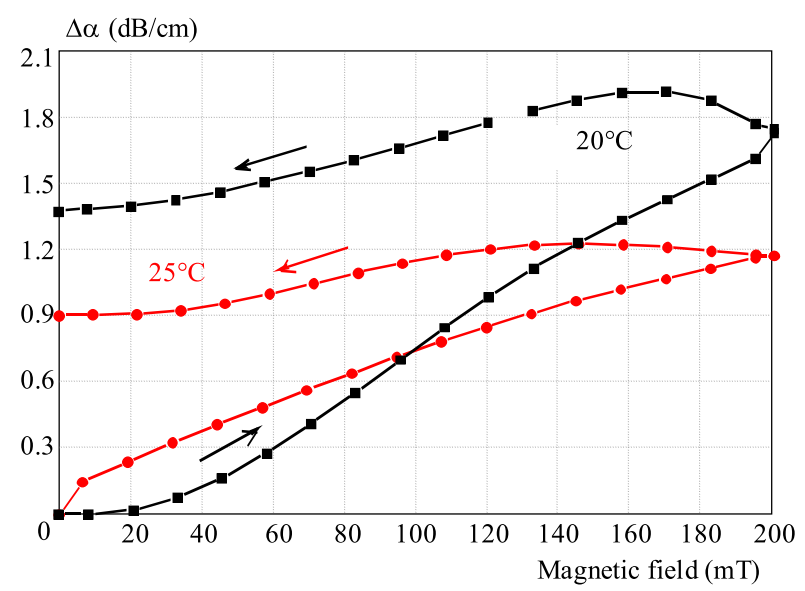

Fig. 2. The dependence of acoustic attenuation changes on external magnetic field for $2.5 \% \mathrm{MF}$ magnetic fluid based on TECHNOL measured at two different temperatures

that were dispersed in transformer oils. The basic properties of $2.5 \%$ MF based on TECHNOL, such as the density and saturation magnetization were $0.917 \mathrm{~g} / \mathrm{cm}^{3}$ and $9.5 \mathrm{mT}$, respectively. These parameters for $1 \% \mathrm{MF}$ based on MOGUL were equal to $0.89 \mathrm{~g} / \mathrm{cm}^{3}$ and $5.6 \mathrm{mT}$. Volume concentrations, average diameter and standard deviation of magnetic particles were determined from vibrating sample magnetometer measurements.

The attenuation of acoustic wave depends on the magnetic field intensity, the rate of its changes and the temperature of magnetic fluid. Figures 2 and 3 present the change of acoustic attenuation as a function of magnetic field $\boldsymbol{B}$ which was parallel to the acoustic wave vector $\boldsymbol{k}$. The magnetic field was linearly increased ( $3.3 \mathrm{mT}$ per minute) to maximum value $200 \mathrm{mT}$ for various temperatures. The magnetic field after reach the maximum value and 1-minute pause decreased at the same rate.

The results summarized in Fig. 2 indicate also the effect of temperature on the processes ongoing in $2.5 \% \mathrm{MF}$ TECHNOL. The measurements show typical change of the acoustic attenuation at linear change of magnetic field. The increase of attenuation with increasing mag-

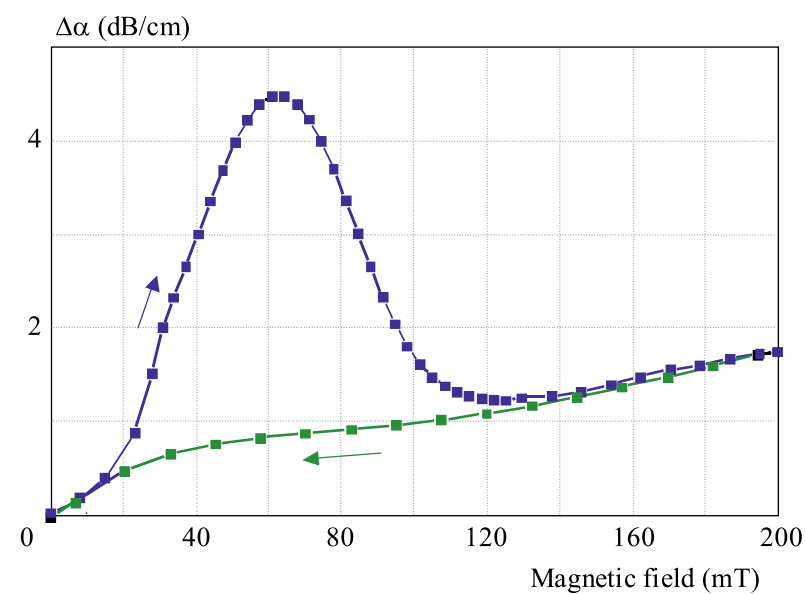

Fig. 3. The dependence of the acoustic attenuation changes on external magnetic field for $2.0 \%$ magnetic fluid based on MOGUL measured at temperature $20^{\circ} \mathrm{C}$

netic field is connected with coupling of nanoparticles to think like shapes - clusters. The processes of clusters creation continue even with decreasing magnetic field. From the value $\sim 160 \mathrm{mT}\left(20^{\circ} \mathrm{C}\right)$ and $\sim 130 \mathrm{mT}\left(25^{\circ} \mathrm{C}\right)$ dominates the process of the thermal degradation of clusters (Brownian motion [10]), which causes the decrease of the acoustic attenuation. With next decreases of magnetic field the coefficient decreases very slowly and it does not return to the initial stage.

Figure 3 shows the acoustic attenuation changes for both increasing and decreasing magnetic field for $2 \% \mathrm{MF}$ based on MOGUL. From the obtained results it can be seen that with increasing magnetic field the acoustic attenuation dramatically increases but only to around $60 \mathrm{mT}$. After reaching the maximum $\Delta \alpha$ decreases up to $120 \mathrm{mT}$ when MF reach the stability and acoustic attenuation $\Delta \alpha$ continues only in slow increase. At decreasing magnetic field there is no maximum, only slow decrease to the initial state. At higher temperatures the development of $\Delta \alpha$ is similar.

To understand the different behaviour observed for magnetic fluids based on TECHNOL and MOGUL, re- 


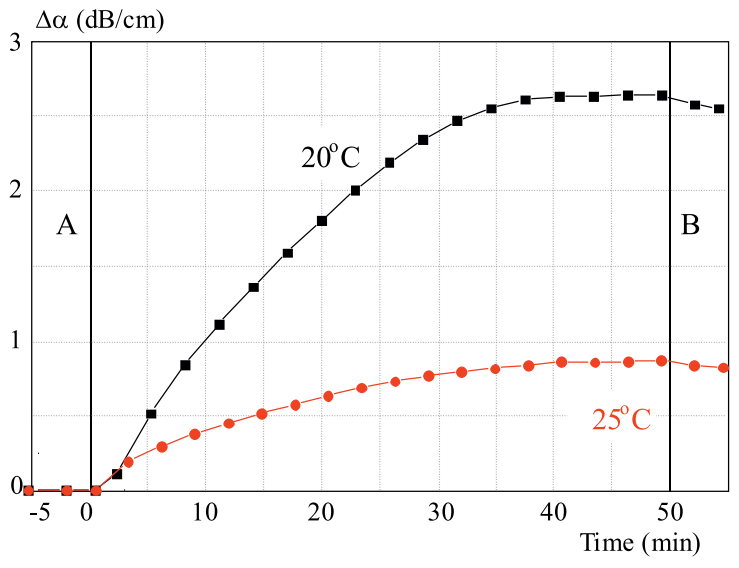

Fig. 4. Experimental data of changes in the acoustic attenuation for jump change of the magnetic field to value $100 \mathrm{mT}$ measured at temperatures $20^{\circ} \mathrm{C}$ and $25^{\circ} \mathrm{C}$ in $2.5 \% \mathrm{MF}$ TECHNOL

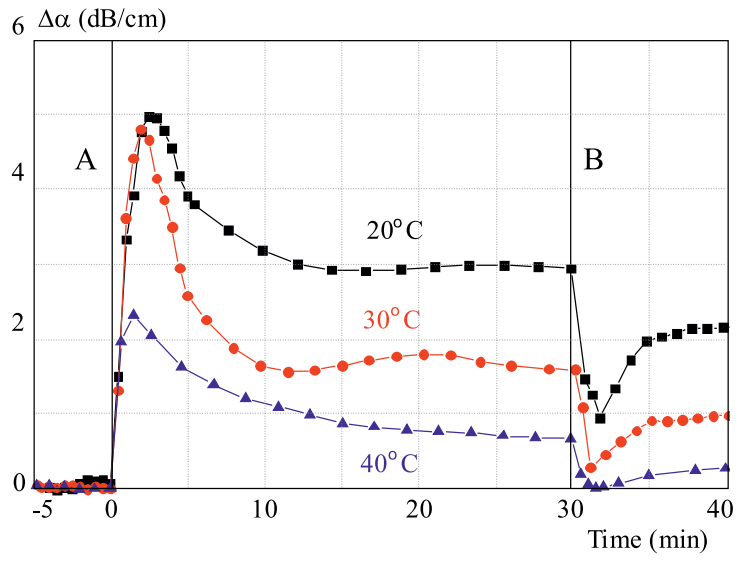

Fig. 5. Experimental data of changes in the acoustic attenuation for jump change of the magnetic field to value $150 \mathrm{mT}$ measured at temperatures $20^{\circ} \mathrm{C}, 30^{\circ} \mathrm{C}$ and $40^{\circ} \mathrm{C}$ in $2 \% \mathrm{MF}$ MOGUL spectively the time development of acoustic attenuation was investigated for jump change of magnetic field. Figures 4 and 5 present the acoustic attenuation changes in the MF subjected to a jumped magnetic field $100 \mathrm{mT}$ for $50 \mathrm{~min}$, $150 \mathrm{mT}$ for $30 \mathrm{~min}$, respectively and its behaviour after removal magnetic field. The acoustic attenuation was measured as a function of time under the following conditions: the magnetic field increased in $10 \mathrm{sec}-$ ond to given constant value (A), then this value of the magnetic field was constant for time and finally the field decreased (B) to zero in 1 second. The area between the lines $\mathrm{A}$ and $\mathrm{B}$ corresponds to the range of time in which the magnetic fluid was subjected to a constant value of the magnetic field.

In the case of MF TECHNOL the change of acoustic attenuation occurred very slowly with increasing time (Fig. 4). It can be seen that only after 40 minute the attenuation reaches constant values in case of temperature $20^{\circ} \mathrm{C}$ and at higher temperature $\left(25^{\circ} \mathrm{C}\right)$ even later. From these results it can be said that the processes of clustering had long timescale. After magnetic field switch off, the change of the acoustic absorption coefficient does not return to initial value and its value almost stationary for long time. This means that the clusters exist also without magnetic field.

The observed changes in $\alpha$ were significant and quite different in $2 \%$ MF MOGUL subjected to the magnetic field. As soon as the magnetic field was applied to the MF the acoustic wave attenuation sharp increased (Fig. 5). This phenomenon was explained by progressing aggregation of magnetic particles into clusters $[2,6,8,10]$. The occurring increase of acoustic attenuation $\alpha$ is the result of additional absorption of the acoustic wave by the spherical clusters formed in the fluid [5]. But after 3 minutes it can be seen dramatic decrease in attenuation. It can be result of collection of clusters to more complicated structure or the process of restructuring of the magnetic fluid. With next increasing time, the value of $\alpha$ reaches a new state of equilibrium. The new state of equilibrium is stable and clusters break up down only after removal of the magnetic field. This behavior coincides with the previous dependence of $\alpha$ on the magnetic field (Fig. 3). After removal of the magnetic field, the acoustic attenuation drastically decreases to the initial value but in time about $5 \mathrm{~min}$ it reaches new equilibrium state. The processes seem to be opposite that when magnetic field was applied. The lifetime of big clusters is in this case very small. In next five minutes acoustic attenuation slowly increases, the fluid structure needs some time to reach new the stable value.

\section{DISCUSSION}

It is known that the interaction between the external magnetic field and the magnetic moment of the nanoparticle in magnetic fluids leads to the aggregation of nanoparticles to new structures $[2,6,8,11]$. These structures enlarge with the magnetic field and this process has the influence on the value of the acoustic attenuation. In our previous works [6,7] or other experimental works $[5,11]$ it was observed that the acoustic attenuation initially increases with increasing magnetic field. This effect can be explained by several parameters. One of is the time constant of creation of higher structures of nanoparticles. Others are temperature or viscosity of given magnetic fluids. However, following progress at higher magnetic field can be different depending on the structure changes caused by developing of cluster shape in individual cases.

The results obtained for $2.5 \%$ MF TECHNOL indicate a significant effect of temperature on the acoustic attenuation. The measurement of the acoustic attenuation at temperature $20^{\circ} \mathrm{C}$ shows also higher hysteresis and large changes originated from the process of aggregation of nanoparticles to dimers and higher oligomers: trimers, tetramers, etc [12]. At higher magnetic field also longer thin chains can arise known as clusters $[4,5,8]$. The process of creation of cluster continues even at decreasing magnetic field. With next decrease of magnetic field the attenuation decreases very slowly. Under this we can say 
that only process leading to the formation oligomers, thin chains or single clusters underway for this magnetic liquid and this type of liquid. This is associated with longer life of clusters for this type of magnetic liquid because they exist although magnetic field is zero. With increasing temperature the thermal motion increases resulting in decrease of numbers of oligomers or clusters and their length. The both smaller numbers of clusters and their shorter length induce the smaller influence on acoustic attenuation at temperature $25^{\circ} \mathrm{C}$. Very long time of creation of thin chains or single clusters is confirmed by measurement after jump change of magnetic field to its constant value. In this case the time response associated with creation of thin chains on the jump change of magnetic field is several tens of minutes. Also decay of these chains is relative long, because they exist also at zero magnetic field.

In the case of $2 \%$ MF MOGUL the situation is rather different. There is big change of acoustic attenuation between 40-100 mT. Similar results were observed by Jozefczak et al [5] with water-based biocompatible MF. This phenomenon can be explained by processing aggregation of magnetic nanoparticles in thin chainlike - clusters. With increasing magnetic field $\Delta \alpha$ increases because of the viscosity of MF increases. The maxima in $\Delta \alpha$ are the result of an additional resonance absorption of ultrasonic wave by the spherical clusters formed in fluid. With next increasing magnetic field the process of clustering continues, but with new important features. Existed clusters connect together forming bigger clusters, what consequently decreases the number of chainlike or smaller clusters. These processes have influence on the value of the acoustic attenuation - dramatic decreases of its value. At $\sim 120 \mathrm{mT}$ this type of $\mathrm{MF}$ reaches a new state of equilibrium in the structure of nanoparticles, that is only slight function of external magnetic field. The case of jump change of magnetic field fully corresponds to previous results. In the first minute after the jump change of magnetic field acoustic attenuation significantly increased. This phenomenon as it was already mentioned can be explained by aggregation of nanoparticles to chain-like clusters that starts over $40 \mathrm{mT}$. In this case the magnetic fluid does not reach some new state but there is following big decrease of $\alpha$ that takes around 3 minutes. In this situation the magnetic field has value $150 \mathrm{mT}$ and we know that from $80 \mathrm{mT}$ new process of clustering is occurred. So that after 10 minutes the studied magnetic fluid reaches the new state of equilibrium. This equilibrium state as well as whole development of structure depends on the temperature of MF based on MOGUL.

\section{CONCLUSION}

The influences of both magnetic field and temperature on the structures of investigated magnetic fluids based on the transformer oil TECHNOL and MOGUL were observed using acoustic spectroscopy. From the presented measurement it can be seen that the effect of external magnetic field on the creation of clusters of nanoparticles is dependent on the type of magnetic fluids. In the case of MF TECHNOL has the process of aggregation of the magnetic nanoparticles into chains and their decay relative long time scale, there are long life of clusters. For MF MOGUL the complicated structures of clusters at magnetic field over $100 \mathrm{mT}$ are created. These structures are than at higher magnetic field almost stable. Measurements also confirmed that the lifetime of these structures or clusters is quite short. The further investigation of the viscosity and density as a function of temperature or acoustic attenuation on of MF with different concentration the magnetic field are necessary to understand all processes in these magnetic fluids.

\section{Acknowledgement}

This work was supported by VEGA projects 1/0624/13 and $2 / 0045 / 13$. The authors wish to thank also for the support to the R\&D operational program Centrum of excellence of power electronics systems and materials for their components, No. OPVaV-2008/2.1/01-SORO, ITMS 2622012003 funded by European Community.

\section{REFERENCES}

[1] ODENBACH, S.: Ferrofluids - Magnetically Controlled Suspensions Colloids and Surfaces A, Phys. Eng. Aspects 217 (2003), 171-178.

[2] SHARIFI, I.-SHOKROLLAHI, H.-AMIRI, S. : Ferite-Based Magnetic Nanofluids Used in Hyperthermia Applications, Journal of Magnetism and Magnetic Materials 324 No. 1 (2012), 903-915.

[3] LEE, J. C. LEE,-W. H.-LEE, S. H.-LEE, S. : Positive and Negative Effects of Dielectric Breakdown in Transformer Oil Based Magnetic Fluids, Materials Research Bulletin 47 No. 10 (2012), 2984-2987.

[4] KÚDELČÍK, J.-BURY, P.-KOPČANSKÝ, P.-TIMKO, M. : Dielectric breakdown in mineral oil ITO 100 based magnetic fluid, Physics Procedia 9 (2010), 78-81.

[5] JÓZEFCZAK, A. Acoustic properties of PEG biocompatible magnetic fluid under perpendicular magnetic field, Journal of Magnetism and Magnetic Materials .

[6] KÚDELČÍK, J.-BURY, P.—DRGA, J.-KOPČANSKÝ, P.ZÁVIŠOVÁ, V.-TIMKO, T. : Comparison of Theories of Anisotropy in Transformer Oil-Based Magnetic Fluids, Advances in Electrical and Electronic Engineering 11, Vol.2 (2013), 147-155.

[7] KÚDELČÍK, J.-BURY, P.—DRGA, J.-KOPČANSKÝ, P.ZÁVIŠOVÁ, V.-TIMKO, T.: Temperature Effect on the Structure of Transformer Oil Based Magnetic Fluids Using Acoustic Spectroscopy, Acta Physica Polonica A 121 No. 5-6 (2012), 1170-1172.

[8] SATOH, A.: Three-Dimensional Monte Carlo Simulations of Internal Aggregate Structures in a Colloidal Dispersion Composed of Rod-Like Particles with Magnetic Moment Normal to the Particle Axis, Journal of Colloid and Interface Science 318 No. 1 (2008), 68-81.

[9] MENDELEV, V. S.-IVANOV, A. O.: Ferrofluid Aggregation in Chains under the Influence of a Magnetic Field, Physics Review E 70 (2004), 051502. 
[10] VASZIOVÁ, G.--TÓTHOVÁ, J.-GLOD, L.-LISÝ, V.: Thermal Fluctuations in Electrical Circuits and the Brownian Motion, Journal of Electrical Engineering 61 No. 4 (2010), 252-256.

[11] SKUMIEL, A.: The Effect of Temperature on the Anisotropy of Ultrasound Attenuation in a Ferrofluid, Phys. D: Appl. Phys. 37 (2004), 3073, doi:10.1088/0022-3727/37/22/003.

[12] MOROZOV, K. I.-SHLIOMIS, M. I. : Ferrofluids: Flexibility of Magnetic Particle Chains, J. Phys.: Condens. Matter 16 (2004), 3807-3818.

\section{Received 15 April 2013}

Jozef Kúdelčík born 1975 in Ružomberok, Slovakia. In 1998 he graduated (Mgr) at the Department of Plasma physics of the Faculty of Mathematics and Physics at UK in Bratislava. He defended his PhD (2003) in the field of stage of breakdown in the mixtures with $\mathrm{SF}_{6}$. In 2011 (doc), he habilitated in Electrotechnology and materials. Since 1998 he has been working as a researcher at the Department of Physics at the University of Žilina. His scientific research is focused on discharge mechanism in gases and in dielectric, as water or oil. In present he is interested about process in magnetic fluid in the external magnetic field studied by acoustic spectroscopy.

Peter Bury received the MSc in experimental physics in 1972 and $\mathrm{PhD}$ in 1982 at the Faculty of Natural Sciences (Faculty of Mathematics and Physics), Comenius University, Bratislava. Currently he is Professor of Physics of Condensed Matter and Acoustics at Department of Physics, Faculty of Electrical Engineering, Žilina University. The most of his research work was orientated on the study of semiconductors and semiconductor structures using acoustic methods. Formerly he investigated $\mathrm{Cr}$ states in GaAs using APR technique, later deep centers in both semiconductor and semicon- ductor structures using acoustic transient spectroscopy (ADLTS). He used methods of acoustic spectroscopy also to study glasses, ferroelectrics and at present also magnetic fluids.

Peter Kopčanský was born in December 1955, in Zakamenne, Slovakia. In 1980 he graduated at the Department of Theoretical Physics of the Faculty of Sciences at Šafarik University in Košice. He defended his PhD (1985) in the field of transport properties of disordered systems like amorphous materials. In 2002 he habilitated in Physics of condensed matter at Faculty of Sciences of Šafarik University in Košice. Since 1995 he has been working as a researcher at the Department of Magnetism Institute of Experimental Physics SAS. His scientific research is focused on the magnetic fluids namely composite systems of liquid crystals doped by magnetic nanoparticles, magnetic drug targeting as well in the field of dielectric properties of magnetic fluids for application in transformer technology also.

Milan Timko was born in September 1954, in Sobrance, Slovakia. In 1977; he graduated (Mgr) at the Department of Experimental Physics of the Faculty of Sciences at UPJŠ in Košice. He defended his $\mathrm{PhD}$ (1984) in the field of the influence neutron radiation on magnetic and electric properties of amorphous materials Fe-Ni-Si-B and Co-Ni-B. Since 1984 he has been working as a scientific worker at the Department of Magnetism at Institute of Experimental Physics SAS. His scientific research is focused on magnetic and hyperthermic properties of biocompatible magnetic fluids. At last more than seven years he is interested also about dielectric properties of transformer oil based magnetic fluids in external magnetic and electric fields.

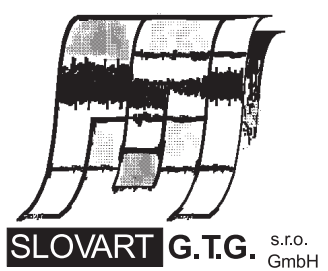

EXPORT - I MPORT
E X P OR T - I M P OR T

of periodicals and of non-periodically printed matters, books and CD-ROMs

Krupinská 4 PO BOX 152, 85299 Bratislava 5, Slovakia tel: ++421 263839 472-3, fax: ++421263839485 info@slovart-gtg.sk; http://www.slovart-gtg.sk

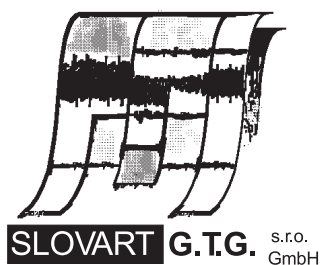

EXPORT - IMPORT 